\title{
FORMALIZED REPRESENTATION FOR THE DATA MODEL WITH THE UNIVERSAL BASIS OF RELATIONS
}

\author{
Vitalii I. Yesin ${ }^{1)}$, Mikolaj Karpinski ${ }^{2)}$, Maryna V. Yesina ${ }^{1)}$, Vladyslav V. Vilihura ${ }^{1)}$ \\ 1) V. N. Karazin Kharkiv National University, 4 Svobody Sq., 61022 Kharkiv, Ukraine \\ v.i.yesin@karazin.ua,m.v.yesina@karazin.ua, viligura93@gmail.com \\ 2) University of Bielsko-Biala, 2 Willowa St., 43-309 Bielsko-Biala, Poland \\ mpkarpinski@gmail.com
}

Paper history:

Received 16 February 2019

Received in revised form 06 September 2019

Accepted 02 December 2019

Available online 31 December 2019

Keywords:

data model;

database.

\begin{abstract}
The goal of the article is to develop a universal (standard) data model that allows you to get rid of the need for a costly policy of doing extra work when developing new ones or transforming existing relational databases (RDBs) caused by dynamic changes in the subject domain (SD). The requirements for the developed data model were formulated. In accordance with the formulated requirements, the data model was synthesized. To simplify the process of creating relational database schemas an algorithm for transforming the description of the subject domain into the relations of the universal basis of the developed model was proposed. The scientific novelty of the obtained results is: a data model that, unlike known ones, allows us to simplify the creation of RDB schemas at the stage of logical design of relational databases, under the conditions of dynamic changes in subject domains, due to the introduced universal basis of relations, as a means of describing structures and the presentation of data for various SDs has been developed.
\end{abstract}

Copyright (C) Research Institute for Intelligent Computer Systems, 2019. All rights reserved.

\section{INTRODUCTION}

In accordance with the existing database design methodology [1-5], the stage of transforming the description of the subject domain (SD) into a database (DB) description, called the logical design stage (the development phase of a logical database schema), follows behind the phase of conceptual modeling. The development of a logical database schema is a rather complex technological process. Neglect of rigorous methods, reliance on heuristic experience and the use of inefficient means is often the main cause of failure in the development of databases of information systems (IS) [1]. At the same time, the use of the existing traditional technology of creating relational databases (RDB), which has become the most widespread on the market of operational database management systems (DBMSs) [6-8], for different areas of company activities in the conditions of a priori uncertainty regarding the demand of individual data in the future and the dynamic changes in the SD, due to the high probability of significant time and financial costs for
DB reengineering is not always acceptable. This caused the need to create new universal models and methods that would reduce the costs of timely creation, modernization of RDBs having the required qualities.

\section{REQUIREMENTS FOR THE MODEL}

Assessing the disadvantages inherent in the existing traditional approach, due to the considerable laboriousness of the procedure for creating a unique database schema for a simulated SD when developing a new $\mathrm{RDB}$, or converting the current RDB, under certain modifications caused by dynamic changes in the SD, it was decided to search for a possible universal (standard) data model. Such data model allows us to get rid of the need for a cost policy of performing unnecessary work. Based on a variety of different analyzed sources, including [9$18]$, and the need to solve this problem, the main requirements for the model being developed are:

- means for structuring data of various dynamically changeable subject domains in a model should be 
some universal basis of relations, the composition and structure of which should be close (consistent) with the set of formal objects of a some data model used in semantic modeling at the conceptual design stage to simplify the process of displaying a conceptual model of the subject domain in the RBD schema and not to lose the semantics of the SD;

-means of model should allow to represent the static and temporal properties of the objects of the simulated SDs, data structures and integrity constraints;

- ease of transition to computer implementation.

\section{MODEL SYNTHESIS}

The development of such model in the form that is presented hereafter was largely made possible by the "object-event" data model [19]. In the structure of the research, the proposed model is its mapping.

When developing the data model with the universal basis of relations, formal objects (the set of $\mathbb{R}$ relations and $\mathbb{F}$ functions) used to represent the basic concepts of the "object-event" model and the types of interaction between them were refined and formalized as corresponding $n$-ary mathematical relations [19]. Some of them are presented below (expressions (1)-(6)).

So the mathematical relation $C$, as a subset of a Cartesian product $\left.C \subseteq C_{1} \times C_{2} \times\left(C_{1} \cup\left\{c_{1}^{\text {null }}\right\}\right)\right) \times M_{1}$, whose extension includes data about classes of objects and their interrelations among themselves for all simulated SDs, is defined by the following expression:

$$
\begin{aligned}
& \left.C \subseteq C_{1} \times C_{2} \times\left(C_{1} \cup\left\{c_{1}^{\text {null }}\right\}\right)\right) \times M_{1}= \\
& =\left\{\left(c_{1}, c_{2}, c_{3}, m_{1}\right) \mid c_{1} \in C_{1} \wedge c_{2} \in C_{2} \wedge\right. \\
& \left.c_{3} \in\left(C_{1} \bigcup\left\{c_{1}^{\text {null }}\right\}\right) \wedge m_{1} \in M_{1}\right\},
\end{aligned}
$$

where $C_{1}$ is the set of object class identifiers; $C_{2}$ is the set of object class names; $M_{1}$ is the set of the SD identifiers; $c_{1}^{\text {null }}$ is the special identifier used when there is no "owner" ("without owner") for the object class $c_{1} \in C_{1}$; tuple element $c_{3} \in\left(C_{1} \cup\left\{c_{1}^{\text {null }}\right\}\right)$ defines the hierarchy of object classes.

The mathematical relation $T$, as a subset of a Cartesian product $T \subseteq T_{1} \times T_{2} \times C_{1}$, whose extension includes data about the types of objects and their relationships with certain classes of objects, is defined by the expression:

$$
\begin{aligned}
T \subseteq T_{1} \times T_{2} \times C_{1}= \\
\quad=\left\{\left(t_{1}, t_{2}, c_{1}\right) \mid t_{1} \in T_{1} \wedge t_{2} \in T_{2} \wedge c_{1} \in C_{1}\right\}
\end{aligned}
$$

where $T_{1}$ is the set of identifiers of object types; $T_{2}$ is the set of object type names.

The mathematical relation $R$, as a subset of the Cartesian product of sets $R_{1}, R_{2},\left(R_{1} \cup\left\{r_{1}^{\text {null }}\right\}\right)$, $M_{1}$, whose extension includes data about sections and their interrelations among themselves for all simulated SDs, is defined by the expression:

$$
\begin{aligned}
& R \subseteq R_{1} \times R_{2} \times\left(R_{1} \cup\left\{r_{1}^{\text {null }}\right\}\right) \times M_{1}= \\
& =\left\{\left(r_{1}, r_{2}, r_{3}, m_{1}\right) \mid r_{1} \in R_{1} \wedge r_{2} \in R_{2} \wedge\right. \\
& \\
& \left.\quad r_{3} \in\left(R_{1} \bigcup\left\{r_{1}^{\text {null }}\right\}\right) \wedge m_{1} \in M_{1}\right\}
\end{aligned}
$$

where $R_{1}$ is the set of identifiers for sections; $R_{2}$ is the set of section names; $r_{1}^{\text {null }}$ is the special identifier used when there is no "owner" ("without owner") for the section $r_{1} \in R_{1}$; tuple element $r_{3} \in\left(R_{1} \cup\left\{r_{1}^{\text {null }}\right\}\right)$ defines the hierarchy of sections.

The mathematical relation $O$, as a subset of a Cartesian product of sets $O_{1}, O_{2},\left(O_{1} \cup\left\{o_{1}^{\text {null }}\right\}\right), R_{1}, T_{1}$, whose extension includes data about instances of objects and their relationships (types of interaction) with certain object types, sections, relationships among themselves, is defined by the following expression:

$$
\begin{aligned}
O \subseteq & O_{1} \times O_{2} \times\left(O_{1} \cup\left\{o_{1}^{\text {null }}\right\}\right) \times R_{1} \times T_{1}= \\
=\left\{\left(o_{1}, o_{2}, o_{3}, r_{1}, t_{1}\right) \mid o_{1} \in O_{1} \wedge o_{2} \in O_{2} \wedge\right. & \\
& \left.o_{3} \in\left(O_{1} \bigcup\left\{o_{1}^{\text {null }}\right\}\right) \wedge r_{1} \in R_{1} \wedge t_{1} \in T_{1}\right\},
\end{aligned}
$$

where $O_{1}$ is the set of identifiers of object instances; $\mathrm{O}_{2}$ is the set of instance names of objects; $o_{1}^{\text {null }}$ is the special identifier used when there is no "owner" for the object instance $o_{1} \in O_{1}$; tuple element $o_{3} \in\left(O_{1} \cup\left\{o_{1}^{\text {null }}\right\}\right)$ defines the hierarchy of object instances.

The mathematical relation $B$, as a subset of the Cartesian product of sets $B_{1}, B_{2}, B_{3}$, $\left(B_{1} \cup\left\{b_{1}^{\text {null }}\right\}\right), E_{1}, O_{1}$, is defined by the expression:

$$
\begin{array}{r}
B \subseteq B_{1} \times B_{2} \times B_{3} \times\left(B_{1} \cup\left\{b_{1}^{\text {null }}\right\}\right) \times E_{1} \times O_{1}= \\
=\left\{\left(b_{1}, b_{2}, b_{3}, b_{4}, e_{1}, o_{1}\right) \mid b_{1} \in B_{1} \wedge b_{2} \in B_{2} \wedge\right. \\
b_{3} \in B_{3} \wedge b_{4} \in\left(B_{1} \cup\left\{b_{1}^{\text {null }}\right\}\right) \wedge \\
\left.e_{1} \in E_{1} \wedge o_{1} \in O_{1}\right\},
\end{array}
$$

where $B_{1}$ is the set of identifiers of event instances; $B_{2}$ is the set of times of the beginning of events; $B_{3}$ is the set of times of the ending of events; $b_{1}^{\text {null }}$ is the special identifier used when there is no "owner" 
(event initiator) for the event instance $b_{1} \in B_{1}$; tuple element $b_{4} \in\left(B_{1} \cup\left\{b_{1}^{\text {null }}\right\}\right)$ defines the hierarchy of event instances.

The extension of the relation (5) is the set of tuples, each of which relates to specific event instance, having relationship with the corresponding object instance and possible relationship with the type of "owner-dependent" interaction with another specific event instance.

Mathematical relation $Y$ as a subset of the Cartesian product of sets $Y_{1}, Y_{2}, B_{1}, X_{1}$, where $X_{1}$ is the set of identifiers of the characteristics of events; $Y_{1}$ is the set of identifiers of the values of the characteristics of events; $Y_{2}$ is the set of values of the characteristics of events, is determined by the expression:

$$
\begin{aligned}
Y \subseteq Y_{1} \times Y_{2} \times B_{1} \times X_{1}=\left\{\left(y_{1}, y_{2}, b_{1}, x_{1}\right) \mid\right. \\
\left.y_{1} \in Y_{1} \wedge y_{2} \in Y_{2} \wedge b_{1} \in B_{1} \wedge x_{1} \in X_{1}\right\} .
\end{aligned}
$$

The extension of the relationship (6) is the set of tuples, each of which relates to specific value of the corresponding characteristic of the event instance.

An analysis of all the mathematical relations obtained in a similar way made it possible to conclude that they are sufficiently simple to implement within the relational model. This can be formalized as an expression:

$$
\chi: M_{r} \rightarrow \operatorname{Rel}(T)
$$

where $\quad M_{r}=\left\{\right.$ name $\left(R_{1}^{\prime}\right), \quad$ name $\left(R_{2}^{\prime}\right), \ldots$, $\left.\operatorname{name}\left(R_{19}^{\prime}\right)\right\}$ is the finite set of names of the obtained mathematical relations, where $R_{1}^{\prime}=C$; $R_{2}^{\prime}=T ; \ldots ; R_{19}^{\prime}=K ; \operatorname{Re} 1(T)$ is relational data model over the binary relation $T \subseteq N \times V ; N$ is a countable set of attribute names mapped to the names of the sets on which the mathematical relations (1)-(6) and like them are defined; $V$ is a countable set of values whose elements are the values of all these attributes $(N \cap V=\varnothing)$.

As a result of such a mapping, we will obtain a finite set of relations of the relational model of different arity (as a finite set of relations of different arity between a finite set of domains):

$$
\mathfrak{R}=\left\{R_{1}, R_{2}, \ldots, R_{19}\right\}
$$

where $R_{i}$ is the $i$-th $(i=1 \ldots 19)$ relation, which is mapping of the corresponding mathematical relation (expressions (1)-(6) and similar to them).

The type of each received $i$-th relation does not depend on the state and the modeled subject domain. It is given by a set of attribute names: $\operatorname{at}\left(R_{i}\right)=\left\{A_{i 1}, A_{i 2}, \ldots, A_{i \alpha_{i}}\right\}$, which is called a relation schema with the name $R_{i}$, or simply the relation schema $R_{i}$ [20]. For explicit indication of a specific type of relation, the following recording form was used: $R_{i}\left(A_{i 1}, A_{i 2}, \ldots, A_{i \alpha_{i}}\right)$.

The set of relations obtained is the universal basis of relations of the model $(\mathfrak{R})$, which can be represented as follows:

$$
\begin{aligned}
& R_{1}\left(A_{11}, A_{12}, A_{13}, A_{14}\right)=C\left(c_{1}, c_{2}, c_{3}, m_{1}\right) \\
& R_{2}\left(A_{21}, A_{22}, A_{23}\right)=T\left(t_{1}, t_{2}, c_{1}\right) \\
& R_{19}\left(A_{19_{1}}, \ddot{A}_{19_{2}}, \ldots, A_{19_{5}}\right)=K\left(k_{1}, k_{2}, j_{1}, o_{1}, v_{1}\right) .
\end{aligned}
$$

A finite set of attribute names:

$$
\operatorname{at}(\mathfrak{R})=\bigcup_{i=1}^{n} \operatorname{at}\left(R_{i}\right)
$$

where $n=19$ and finite set of domains: $D=\left\{D_{1}, D_{2}, \ldots D_{m}\right\} \quad$ are associated with the universal basis of relations $\mathfrak{R}$ (9), which is an invariant with respect to various SDs. Where for each $D_{l}$ there is an attribute name $A_{i \alpha_{j}} \in \operatorname{at}(\mathfrak{R})$ such as $D_{l}=\operatorname{dom} A_{i \alpha_{j}}$, where $l=1 . . m$, and $j=1 . . n$.

The diagram of the universal basis of relations of the model in IDEF1X notation is represented in Fig. 1.

In accordance with the definition of the data model, considering the above expressions and notation for the basis of relations, the proposed data model in a formalized form can be represented as the tuple:

$$
\mathrm{M}_{\mathrm{ubr}}=\langle\mathfrak{R}, \operatorname{Pr}, \mathrm{L}\rangle
$$

where $\mathfrak{R}$ is the universal basis of relations of the model (9); $\mathrm{Pr}$ is a set of integrity constraints; $\mathrm{L}$ is data manipulation language.

A set of integrity constraints $\mathrm{Pr}$. In the proposed model it specifies both integrity constraints inherent and supported by the relational data model, within which it is implemented, and the restrictions inherent for the "object-event" data model, the mapping of which it is. 


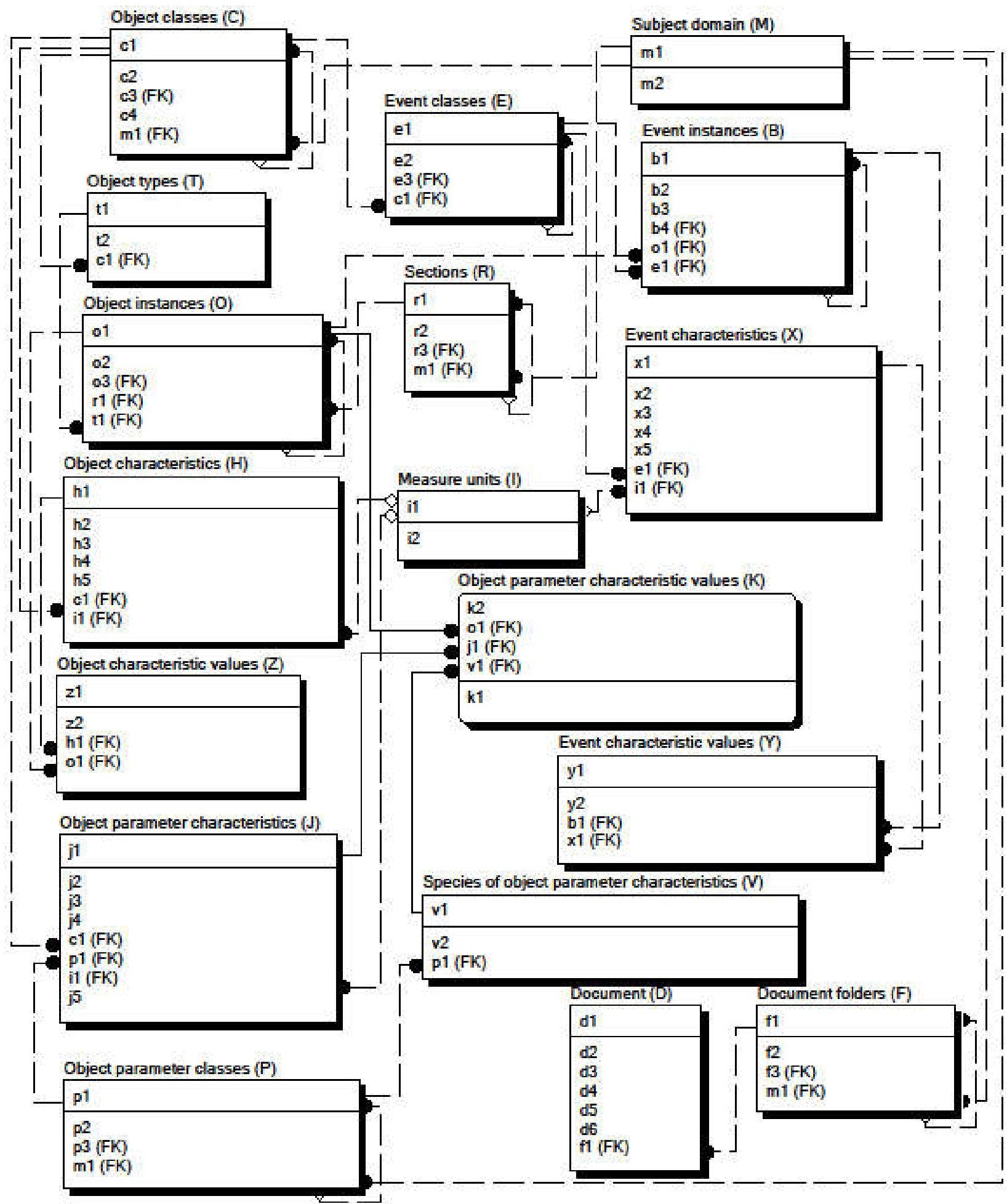

Figure 1 - Diagram of the universal basis of relations of the model

Some of them are given below:

- entity integrity rule: $\operatorname{Pr}_{P K} \in \operatorname{Pr}$ (in relations $\mathfrak{R}$, no attribute of the primary key can contain null (missing) values, denoted as null, and have the property of irreducibility);

-rule of referential integrity or foreign key integrity: $\operatorname{Pr}_{F K} \in \operatorname{Pr}\left(R_{i}\left[K_{F K}\right] \subseteq R_{j}\left[K_{P K}\right], K_{P K}\right.$ is primary key (primary key attributes); $K_{F K}$ is foreign key (foreign key attributes); designations $R_{i}\left[K_{F K}\right], R_{j}\left[K_{P K}\right]$ are used for the projection $R_{i}$ on $K_{F K}, R_{j}$ on $K_{P K}$ );

-domain constraints: $\operatorname{Pr}_{d o m} \in \operatorname{Pr} \quad$ (including $\operatorname{Pr}_{\text {not_null }} \in \operatorname{Pr}$ is required data (not null data); special domains due to the features of the proposed model: $\operatorname{dom}\left(h_{4}\right)=\left\{F^{\prime},{ }^{\prime} P^{\prime}\right\}$ is domain of features 
of belonging the characteristic to one of the types [19]: object type characteristic - ' $P$ ', factual characteristic of the object - $' F^{\prime}$; $\operatorname{dom}\left(j_{4}\right)=\left\{H^{\prime},{ }^{\prime} D^{\prime},{ }^{\prime} M{ }^{\prime},{ }^{\prime} Q^{\prime},{ }^{\prime} Y^{\prime},{ }^{\prime} A '\right\} \quad$ is domain of features of the object parameter measurement interval; domain of the relation names $\mathfrak{R}: \operatorname{dom}\left(w_{1}\right)=\left\{\right.$ name $\left(R_{1}\right)$, name $\left(R_{2}\right)$, name $\left(R_{3}\right)$, ...\}; domain of data types of characteristics for objects, events, object parameters: $D_{U D M}=\{$ 'boolean', 'char', 'number', 'date' $\}$;

domains of the characteristics of objects, events, object parameters belonging to the enumerated (list) type: $\quad D_{C h O}^{i}=\operatorname{dom}\left(h_{2}^{i_{\text {ist }}}\right) ; \quad D_{C h E}^{j}=\operatorname{dom}\left(x_{2}^{j_{\text {list }}}\right)$; $D_{C h P O}^{k}=\operatorname{dom}\left(j_{2}^{k_{\text {list }}}\right)$; domain of units of physical quantities of characteristics of objects, events, object parameters for the simulated SD: $\left.\operatorname{dom}\left(i_{2}\right)\right)$;

-constraints that simplify the specification of the so-called corporate integrity constraints (general constraints):

a) constraints on the maximum number of object instances for a certain object class:

$$
\begin{aligned}
C=\{ & \left(c_{1}, c_{2}, c_{3}, c_{4}, m_{1}\right) \mid c_{1} \in C_{1} \wedge c_{2} \in C_{2} \wedge \\
& \left.c_{3} \in\left(C_{1} \bigcup\left\{c_{1}^{\text {null }}\right\}\right) \wedge c_{4} \in \mathbb{N}^{+} \wedge m_{1} \in M_{1}\right\},
\end{aligned}
$$

where $c_{4}$ is the element of the ordered five of the relation (12) defined on the set of positive natural numbers $\mathbb{N}^{+}$. It is used to limit the number of instances of objects $\left(\left|O_{1}\right| \leq c_{4}\right)$ for the object class specified by the element of the relation tuple (12).

b) constraints on the maximum number of values that can be assigned to a certain event characteristic for an event instance of a specified class:

$$
\begin{array}{r}
X=\left\{\left(x_{1}, x_{2}, x_{3}, x_{4}, x_{5}, e_{1}, i_{1}\right) \mid x_{1} \in X_{1} \wedge\right. \\
x_{2} \in X_{2} \wedge x_{3} \in X_{3} \wedge x_{4} \in X_{4} \wedge x_{5} \in \mathbb{N}^{+} \wedge \\
\left.e_{1} \in E_{1} \wedge i_{1} \in\left(I_{1} \bigcup\{\omega\}\right)\right\},
\end{array}
$$

where $x_{5}$ is the element of the ordered seven of the relation (13) defined on the set $\mathbb{N}^{+}$. It is used to limit the number of values that can be assigned to an event characteristic specified by the element $x_{1}$ of the relation tuple (13) for an instance of the event class $E_{1}$ specified by the element $e_{1}$.

c) constraints on events (with one object instance in the same time interval only one event of the same class can occur):

$$
\begin{gathered}
B=\left\{\left(b_{1}, b_{2}, b_{3}, b_{4}, e_{1}, o_{1}\right) \mid b_{1} \in B_{1} \wedge b_{2} \in B_{2} \wedge\right. \\
b_{3} \in B_{3} \wedge b_{4} \in\left(B_{1} \bigcup\left\{b_{1}^{\text {null }}\right\}\right) \wedge e_{1} \in E_{1} \wedge \\
o_{1} \in O_{1} \wedge\left(\forall b_{1} \forall b_{2} \forall b_{3} \forall b_{4} \forall e_{1} \forall o_{1}\right. \\
\left(\forall b_{1}^{\prime} \in B_{1}\right)\left(\forall b_{2}^{\prime} \in B_{2}\right)\left(\forall b_{3}^{\prime} \in B_{3}\right) \\
\left(B_{p r}\left(b_{1}, b_{2}, b_{3}, b_{4}, e_{1}, o_{1}\right) \wedge\right. \\
B_{p r}\left(b_{1}^{\prime}, b_{2}^{\prime}, b_{3}^{\prime}, b_{4}, e_{1}, o_{1}\right) \rightarrow\left(\left(\neg \left(\left(b_{2} \leq b_{2}^{\prime}\right) \wedge\right.\right.\right. \\
\left.\left.\left(b_{3} \geq b_{3}^{\prime}\right)\right) \wedge\left(b_{2} \leq b_{3}\right) \wedge\left(b_{2}^{\prime} \leq b_{3}^{\prime}\right)\right) \vee \\
\left.\left.\left.\left.\left(\left(b_{2} \neq b_{2}^{\prime}\right) \wedge\left(b_{3}=\omega\right) \wedge\left(b_{3}^{\prime}=\omega\right)\right)\right)\right)\right)\right\},
\end{gathered}
$$

where $\mathrm{B}_{p r}\left(b_{1}, b_{2}, b_{3}, b_{4}, e_{1}, o_{1}\right), \quad B_{p r}\left(b_{1}^{\prime}, b_{2}^{\prime}, b_{3}^{\prime}, b_{4}\right.$, $\left.e_{1}, o_{1}\right)$ are predicates (predicate symbols) matching the relation $B$, etc.

A widespread and important type of integrity constraints are functional dependencies between two sets of attributes of specified relation [21]. In the consideration case, functional dependencies specify certain types of constraints imposed on relation $\mathfrak{R}$ associated with the features of their structure and purpose.

Thus, the given above expressions (12)-(14); constraints of entity integrity $\left(\operatorname{Pr}_{P K}\right)$ and referential integrity $\left(\operatorname{Pr}_{F K}\right)$; constraints $\operatorname{Pr}_{\text {not_null }}$; domain constraints $\left(\mathrm{Pr}_{\text {not_null }}\right)$; functional dependencies constitute the set of integrity constraints $\operatorname{Pr}$ of formula (11).

In the manipulative component of the proposed model (element L in (11)) two ways of operating with data are defined. Namely, the basic operations of data model with the universal basis of relations (as well as the relational model on the basis of which it is implemented) can be expressed both using the language of relational algebra based on set theory, and using the language of relational calculus based on the logic of the predicate calculus of first order.

\section{MAPPING A CONCEPTUAL MODEL OF SUBJECT DOMAIN INTO THE RELATIONS OF UNIVERSAL BASIS}

To simplify the process of creating logical schemas of RDBs under the conditions of dynamic changes in subject domains and limited time and financial resources, an algorithm was developed to transform the description of the SD executed by means of the "object-event" data model to the relations of universal basis of the proposed data model. A block diagram of this algorithm is shown in Fig. 2.

The elements of the sets of corresponding formal objects correlated with the basic concepts of the "object-event" model [19], which are used to represent the conceptual schema of the SD, find their mapping in the values of the corresponding attributes of the relations of universal basis of the 
proposed data model, firstly, in the relations associated with the metadata, and then with the data of the modeled SD. The result of such mapping is a logical schema of created RDB, which has a universal structure for representing the static and temporal properties of objects of various dynamically changing SDs.
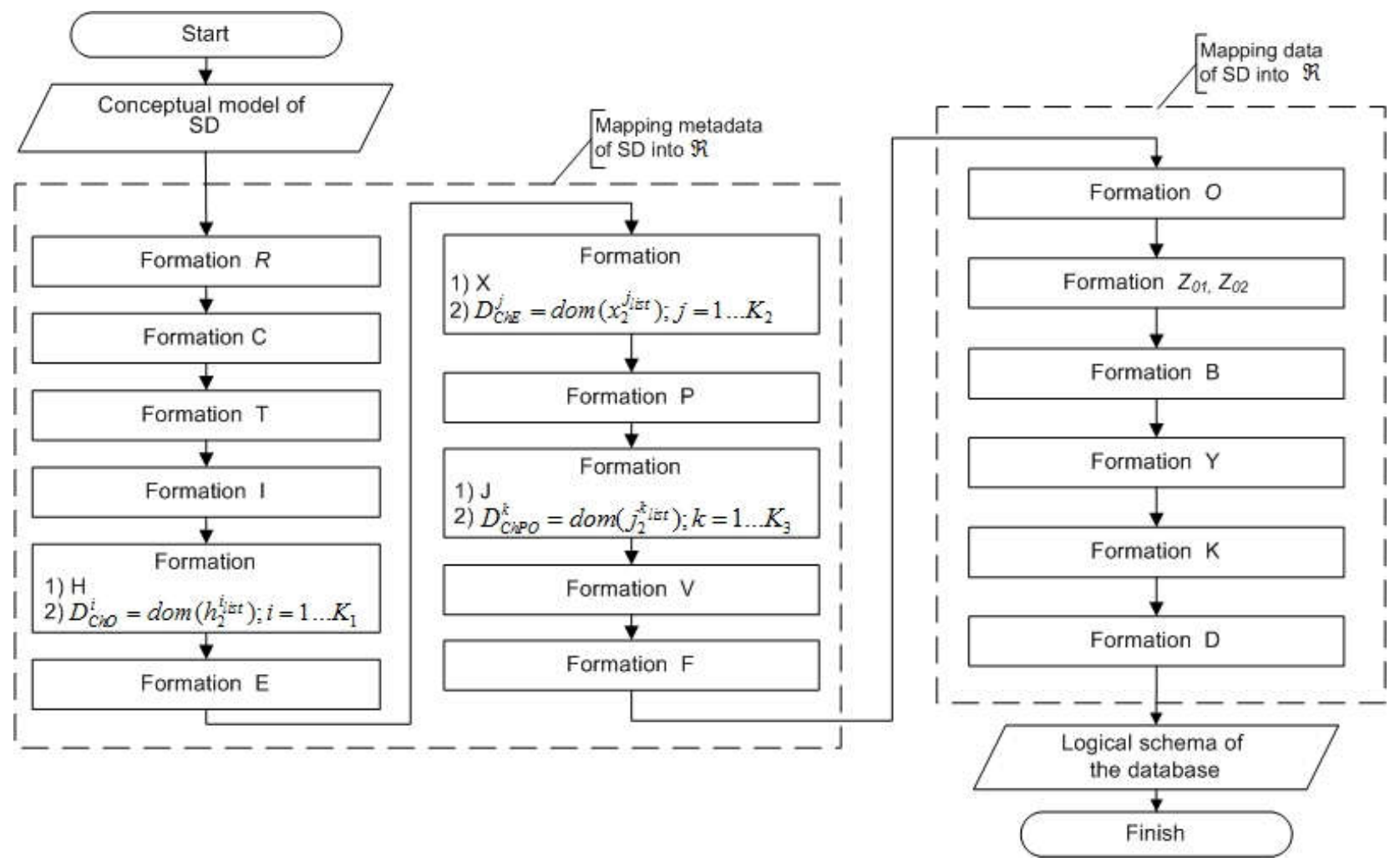

Figure 2 - Block diagram of the algorithm for transforming the description of SD into the relations of the universal basis

In this approach, the universal basis of relations at the logical design stage of RDB is used to describe both data structures (schemas in the traditional sense) and the presentation of the data itself of various dynamically changing subject domains, simplifying the further presentation of the conceptual model of the SD in a computer environment.

In fact, the proposed approach to the creation (formation within the framework of the universal basis of relations) of the RDB logic schema turns the process of transforming various conceptual models of the SD into a solution of the same type task with different options of conditions, thereby saving general time and effort (staff months), both for developing new and modernization of existing databases. There is no need to re-create each time a unique logical, and subsequently physical schema when developing a new database, or substantially transform them during modernization.

The conducted estimate of the effectiveness of the development process, the modernization of RDBs built based on the data model with the universal basis of relations in the conditions of dynamic changes of the SD showed the advantage of the proposed approach over the traditional technology for designing relational databases. Namely, through using technology of creating databases with the universal basis of relations, it is possible to achieve possible savings in effort - up to $60 \%$ and time - up to $25 \%$ compared to traditional technology for designing RDBs. The reliability of the estimates of the achieved advantages has been experimentally confirmed. So, for example, when developing a database for the information system of a trucking company, thanks to the use of the proposed technology, which is based on the data model with the universal basis of relations, efforts were reduced by about $55 \%$, and time - by $20 \%$ relative to the characteristics of a similar project implemented in the framework of the traditional technology for designing RDBs.

\section{CONCLUSIONS}

The scientific novelty of the obtained results is:

1. A new data model has been developed, the main components of which are:

- universal basis of relations, resulting from the transformation of a set of formal objects of the "object-event" model;

-a set of integrity constraints. In the model both integrity constraints inherent and supported by the 
relational data model are specified, on the basis of which it is implemented, and the constraints inherent for the "object-event" data model, the mapping of which it is (domain constraints, constraints that simplify the specification of corporate integrity constraints (constraints of the SD), etc.);

-the manipulative component, which, as in the relational model, provides two mechanisms for operating with data: using the language of relational algebra; using the language of relational calculus.

The proposed data model is the tool that at the stage of logical design of relational databases, under the conditions of dynamic changes of SDs, allows you to simplify the creation of database schemas due to the developed universal basis of relations, as a means of describing the structures and data representation of various dynamically changing subject domains.

2. An algorithm for transforming the description of a subject domain executed by means of the "object-event" data model to the relations of universal basis proposed data model, simplifying the process of creating logical schemas of relational databases under the conditions of dynamic changes of the SDs and limited time and financial resources has been developed.

\section{REFERENCES}

[1] T. M. Connolly, C. E. Begg, Database Systems: A Practical Approach to Design, Implementation, and Management, sixth edition, Harlow, Essex, England, Pearson Education Limited, 2015, 1329 p.

[2] E. Z. Zinder, "Database design: new requirements, new approaches," $D B M S$, vol. 3, pp. 10-22, 1996. (in Russian).

[3] V. V. Pasichnyk, V. A. Reznichenko, Organization of Databases and Knowledge, BHV Publishing Group, Kyiv, 2006, 384 p. (in Ukrainian).

[4] D. C. Tsichritzis, F. H. Lochovsky, Data Models, Finance and Statistics, Moscow, 1985, 344 p. (in Russian).

[5] S. D. Kuznetsov, Database Basics, Internet University of Information Technologies, BINOM, Laboratory of Knowledge, Moscow, 2007, 484 p. (in Russian).

[6] DB-Engines Ranking, 2018 [Online]. Available at: https://db-engines.com/en/ranking.

[7] M. Adrian, D. Feinberg, N. Heudecker, Magic Quadrant for Operational Database Management Systems, 22 October 2018, ID G00346575, 2018. [Online]. Available at: https://www.gartner.com/doc/reprints?id=15LPN68I\&ct $=181015 \& \mathrm{st}=\mathrm{sb}$.
[8] D. Feinberg, M. Adrian, N. Heudecker, Critical Capabilities for Operational Database Management Systems, 23 October 2018, ID G00353669, 2018. [Online]. Available at: https://www.gartner.com/doc/reprints?id=15LPN68L\&ct $=181015 \& \mathrm{st}=\mathrm{sb}$.

[9] P. A. Bernstein, U. Dayal, D. J. DeWitt, et al., "Future directions in DBMS research - The Laguna Beach Participants," ACM SIGMOD Record, vol. 18, issue 1, pp. 17-26, 1989.

[10] P. A. Bernstein, M. L. Brodie, S. Ceri, et al., "The asilomar report on database research," ACM SIGMOD Record, vol. 27, issue 4, pp. 7480, 1998.

[11] Yu. A. Pergamentsev, "Database design based on the universal data model," Proceedings of the conference on Corporate Databases 2002, Moscow, Russia, April 16-17, 2002, [Online]. Available at: http:/citforum.ru/seminars/ cbd2002/111.shtml. (in Russian).

[12] E. D. Vyazilov, A. A. Fedortsov, "Universal data storage model taking into account the life cycle of objects," Proceedings of the Sixth AllRussian Open Annual Conference "Modern Problems of Remote Sensing of the Earth from Space", Moscow, Russia, November 10-14, 2008, [Online]. Available at: http://d902.iki. rssi.ru/theses-cgi/thesis.pl?id=1197. Russian).

[13] E. D. Vyazilov, A. A. Fedortsov, A. E. Kobelev, "Unification of data structure for field research, exploration and resources using of World Ocean," Proceedings of the 10-th AllRussian Scientific Conference "Electronic libraries: promising methods and technologies, electronic collections", RCDL'2008, Dubna, Russia, October 7-11, 2008, pp. 293-298. (in Russian).

[14] V. Filatov, V. Radchenko, "Reengineering relational database on analysis functional dependent attribute", Proceedings of the X Intern. Scient. and Techn. Conf. "Computer Science \&amp; Information Technologies» (CSIT'2015), Lviv, Ukraine, September 14-17, 2015, pp. 85-88.

[15] D. Abadi, R. Agrawal, A. Ailamaki, M. Balazinska, P. A. Bernstein, M. J. Carey, S. Chaudhuri, J. Dean, A. Doan, M. J. Franklin, J. Gehrke, L. M. Haas, A. Y. Halevy, J. M. Hellerstein, Y. E. Ioannidis, H. V. Jagadish, D. Kossmann, S. Madden, S. Mehrotra, T. Milo, J. F. Naughton, R. Ramakrishnan, V.r Markl, C. Olston, B. C. Ooi, C. Re, D. Suciu, M. Stonebraker, T. Walter, J. Widom, "The Beckman report on database research," $A C M$ SIGMOD Record, vol. 43, issue 3, pp. 61-70, 2014. 
[16] H. Garcia-Molina, J. D. Ullman, J. Widom, Database Systems. The Complete Book, 2th ed., Pearson Prentice Hall, 2009, 1203 p.

[17] O. O. Varlamov, Evolutionary Databases and Knowledge for Adaptive Synthesis of Intelligent Systems. Mivar Information Space, Radio and Communication, Moscow, 2002, 282 p. (in Russian).

[18] V. I. Yesin, M. V. Yesina, S. G. Rassomakhin, M. Karpinski, "Ensuring database security with the universal basis of relations," in: K. Saeed, W. Homenda (Eds.) Computer Information Systems and Industrial Management. CISIM 2018. Lecture Notes in Computer Science, vol. 11127, Springer, Cham, 2018, pp. 510-522.

[19] V. I. Yesin, M. V. Yesina, "Means for conceptual modeling of information system databases," in: S. G. Rassomakhin, A. A. Kuznetsov (Eds.), Security and noise immunity of telecommunication systems: new solutions to the codes and signals design problem, ASC Academic Publishing, Minden, Nevada, 2017, pp. 160-196.

[20] M. Sh. Tsalenko, Modeling Semantics in Databases, Moscow, Russia, Science, Main Edition of the Physical and Mathematical Literature, 1989, 288 p. (in Russian).

[21] C. J. Date, An Introduction to Database Systems, 8th Edition, Pearson, AddisonWesley, 2004, 983 p.

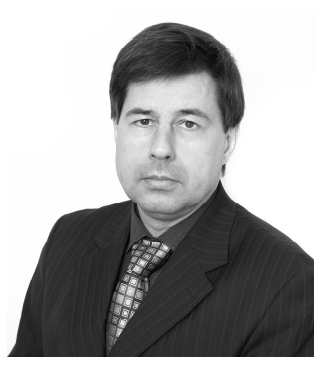

Vitalii Yesin, Doctor of Engineering Sciences, specialty "Information Technologies". Professor of the Security of Information Systems and Technologies Department of the Computer Science Faculty of the V. N. Karazin Kharkiv National University.
Research interests: models and methods for developing information systems databases and ensuring their security.

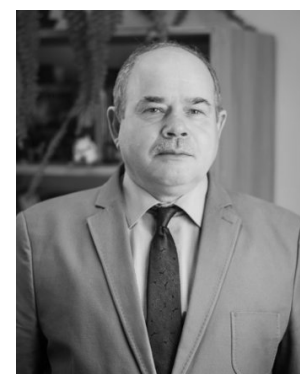

Mikołaj Karpiński, Doctor of Science Electrical and Magnetic Instrumentation, Full Professor in Security of Information Technologies, Head of the Department of Informatics and Automatics of University of Bielsko-Biala.

Research interests: cybersecurity, cryptographic security, computer systems.

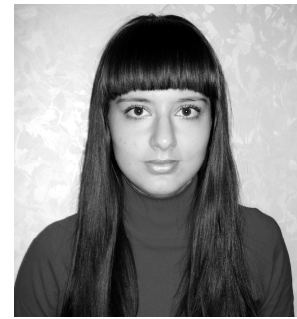

Maryna Yesina, PhD of Engineering Sciences, specialty "Information Protection Systems", Senior Lecturer of the Security of Information Systems and Technologies Department of the Computer Science Faculty of the V. N. Karazin Kharkiv

National University.

Research interests: cybersecurity, cryptographic security, post-quantum secure algorithms.

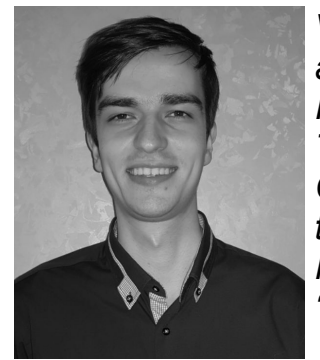

Vladyslav Vilihura, Postgraduate student of the Security of Information Systems and Technologies Department of the Computer Science Faculty of the V.N. Karazin Kharkiv National University, specialty "Cybersecurity".

Research interests: databases and ensuring their security. 